\title{
La sociología de la educación y la teoría del reconocimiento de Axel Honneth
}

\section{The sociology of education and the recognition theory of Axel Honneth}

\author{
Mauricio Rebelo Martins, Francesc J. Hernàndez y Benno Herzog'
}

\section{Resumen}

El artículo relaciona la teoría del reconocimiento de Axel Honneth y la sociología de la educación. Hace una exposición breve de las formulaciones y reformulaciones de la teoría del reconocimiento y explica las relaciones de la educación con los modos de reconocimiento. El artículo critica las perspectivas dialógicas y comenta las posibilidades que puede ofrecer esta teoría para el análisis de las prácticas educativas. Por último, se introduce la noción de reconocimiento anticipatorio y se relaciona la teoría con los procesos de aprendizaje y con la noción de semiformación.

\section{Palabras clave}

Teoría del reconocimiento, Axel Honneth, Escuela de Fráncfort, Jürgen Habemas.

\section{Abstract}

The article relates the recognition theory of Axel Honneth and the sociology of education. It gives a brief exposition of the formulations and reformulations of the theory of recognition and explains the relations of education with modes of recognition. The article criticizes the dialogical perspectives and discusses the possibilities that this theory can offer forthe analysis of educational practices. Finally, the notion of anticipatory recognition is introduced and the theory is related to the learning processes and to the notion of semiformation.

\section{Key words}

Recognition theory, Axel Honneth, Frankfurt School, Jürgen Habemas. 


\section{Introducción}

Cuando en octubre de 2016, la Academia Sueca comunicó la concesión del Premio Nobel al cantante Bob Dylan, algunas personas recordamos que, diez años antes, Axel Honneth le había dedicado un congreso como director del Instituto de Investigación Social (IIS) de la Universidad de Fráncfort del Meno (Honneth, Kemper y Klein, 2007). El IIS es la sede de la denominada Escuela de Fráncfort. Efectivamente, la obra de Axel Honneth ha puesto en el orden del día sociológico asuntos relevantes y muy diversos, en el marco de una propuesta ambiciosa conocida como la teoría del reconocimiento. Esta teoría ha alcanzado recientemente una formulación madura, con lo que podríamos denominar en cierto sentido «trilogía» en torno a El derecho de la libertad, sin embargo, poco espacio en tan amplia obra se ha dedicado a la educación. En este artículo exploraremos la relación entre esa formulación madura y la sociología de la educación en asuntos como la crítica a las orientaciones dialógicas, la relación del elemento democrático y el meritocrático en la educación, la reconsideración de temas clásicos relacionados con al perspectiva de género, la relación de la educación con asuntos estéticos, la crítica a las tendencias privatizadoras, etc.

En primer lugar, haremos una exposición muy sucinta del estado actual de la teoría del reconocimiento (apartado 2). En segundo lugar, vincularemos esa situación con la sociología de la educación (apartado 3) para extraer, en tercer lugar, algunas conclusiones (apartado 4). Hemos de advertir que, cuando estén traducidas, citaremos las obras de Honneth y otros autores por su versión castellana más reciente. Somos conscientes que hemos incluido en nuestro artículo largas citas de una entrevista autorizada a Honneth, lo que justificamos por su carácter inédito y por el interés de sus palabras.

\section{De la Lucha por el reconocimiento a la trilogía de El Derecho a la Libertad}

La teoría del reconocimiento de Axel Honneth se formula y reformula en dos ciclos bibliográficos. Se formula en La lucha por el reconocimiento (Honneth, 1997), publicada en 1992, obra que comienza a fraguarse por lo menos una década antes, como acredita el siguiente pasaje del artículo «Conciencia moral y dominación de clase» de 1981: «Mi suposición es que la teoría social de Habermas está constituida de manera tal que tiene que ignorar sistemáticamente todas las formas de crítica social existentes que no sean reconocidas por el espacio público político-hegemónico». (Honneth 2000: 57). Este es el punto en el que Honneth rompe con la teoría de la acción comunicativa de Jürgen Habermas, porque, según esta, la crítica social se puede realizar a partir de la aportación normativa que puede deducirse a priori del horizonte de entendimiento inherente a cualquier acción comunicativa. Sin embargo, si se toman en consideración teorías como las de Foucault o Bourdieu, resulta inaceptable postular aquel horizonte de entendimiento, o con los términos de Honneth citados: el espacio público ya está políticamente hegemonizado. Diciéndolo a la manera kantiana, «lo social» despertó a Honneth del sueño pragmáticouniversal habermasiano. De cómo «lo social» ponía en dificultades el proyecto de la Teoría Crítica da cuenta la edición ampliada de su tesis doctoral (Honneth 2009a), sin embargo, la salida a esta aporía exigía un esfuerzo conceptual mayor, que es el que Honneth acomete para su tesis de habilitación: La lucha por el reconocimiento.

Prácticamente la primera mitad del La lucha por el reconocimiento, se dedica a un comentario históricofilosófico sumamente especializado de los escritos juveniles de Hegel, anteriores a su primera gran obra La fenomenología del espiritu (Hegel, 2009), con el que Honneth construye una clasificación de los modos de reconocimiento intersubjetivo (y sus manifestaciones emocionales, cognitivas o sociales). En la segunda mitad, glosa la clasificación con el comentario de autores heterogéneos (como, por ejemplo, Mead o Sorel). Por ello, más que entrar en el detalle del libro, ofreceremos una visión panorámica de qué hace realmente el autor con esta obra. 


\section{Mauricio Rebelo Martins, Francesc J. Hernàndez y Benno Herzog. La sociología de la educación... RASE 2017. Vol. 10. N. ${ }^{\circ}$ 1: Págs. 80-89 doi: http://dx.doi.org/10.7203/RASE. 10.1.9451}

Honneth toma en serio dos afirmaciones correlativas de Theodor W. Adorno en su Dialéctica negativa: «el momento corporal recuerda al conocimiento que el sufrimiento no debe ser» y existen «formas interiores de reflexión» del sufrimiento físico (Adorno: 2011: 191 y 192). Es decir, en la «expresión del sufrimiento» (Adorno 2014: 158) ${ }^{2}$ se concitan inmediatamente aquellos dos elementos a los que aspiraba Habermas: disponer de un principio normativo que permita la crítica social y obtener una cierta racionalidad (una reflexión) que sirva de contrapeso a la tendencia a la barbarie de la razón administrada. O dicho de otro modo: la expresión del sufrimiento de la víctima, aunque no pueda formularse en el espacio público por estar políticamente hegemonizado, ya resulta «público», por cuanto no existen lenguajes privados (Adorno no reconoce explícitamente, que sepamos, esta aportación del segundo Wittgenstein), y representa una «gramática moral» con la que es posible enjuiciar las formas sociales, es decir, hacer teoría crítica de la sociedad.

A partir de esta obra, a nuestro juicio, Honneth se concentró menos en caracterizar las tres formas de reconocimiento que había identificado a partir de los textos de Hegel, a saber, el amor, el derecho y la solidaridad, y más en mostrar las consecuencias de su desconsideración, a saber, la «invisibilidad», la «reificación», las «patologías sociales», el «agravio moral» o, simplemente, «desprecio» (cf. Honneth 2003; 2007; 2009b, 2009c y 2011). Con ello, se ganaba en virtualidad sociológica de la nueva teoría, pero no se abordaba un asunto de fondo relevante. Honneth acepta una línea de crítica importante a su teoría, a saber, la dificultad de deslindar pretensiones de reconocimiento auténticas de otras inauténticas o, en sus palabras, «ideológicas», ya que tanto unas como otras pueden estar originadas en indiscernibles pugnas por no ser reconocidos de sujetos que sufren (sobre la noción de pugna, véase Herzog; Hernàndez, 2012). A partir de su debate con Nancy Fraser sobre la justicia, titulado «¿Redistribución o reconocimiento?» (Fraser \& Honneth 2006), Honneth cobra conciencia que dificultades como la mencionada están ancladas en el hecho de que su clasificación de los modos de reconocimiento mantiene un fundamento antropológico. Por ello emprende una reformulación que asiente la teoría del reconocimiento sobre otra base.

Esta reformulación arranca de una relectura de la Filosofía del Derecho de Hegel. A pesar de este nombre singular, esta obra de Hegel no se aleja mucho de lo que había intentado Kant con su Metafísica de las costumbres o estaba haciendo simultáneamente Comte: establecer una ciencia de la sociedad. No hay que olvidar que el propio Marx comenzó con una revisión de la Filosofía del Derecho la elaboración de su crítica de la economía política (Marx, 1978). Los dos autores, Marx y Honneth, coinciden en la pretensión de mantener el núcleo crítico (dialéctico) de la obra, sin asumir su concepción idealista de la historia. La diferencia radica en la base desde la que acometen el asunto central de la eticidad ${ }^{3}$ : Marx desde la teoría del Estado, como culminación de la dialéctica de la sociedad civil; Honneth desde sus partes previas: el derecho abstracto y la moralidad. O dicho en términos más sociológicos, Marx se acerca al ideal de una sociedad a resguardo de críticas desde un análisis de un Estado que, como el prusiano, había sofocado los anhelos revolucionarios; Honneth desde un estudio de las patologías de la libertad, que surgen precisamente por entenderla de un modo incompleto. Esta elaboración ocupa diversos textos sucesivos de Honneth $(1999,2001,2010,2016)$ que constituyen el núcleo conceptual de lo que ha desarrollado en una obra más divulgativa, aunque extensa: El derecho de la libertad (Honneth, 2014). En síntesis, los modos de reconocimiento no están anclados en disposiciones antropológicas, sino que han ido cristalizando en

2 La definición de Adorno de la misión de la filosofía en este texto, a saber, «llevar al lenguaje el sufrimiento del mundo», bien podría aplicarse también a la sociología.

3 Sobre la relación entre eticidad y educación, cf. Hernàndez 2009. 
instituciones sociales. Estas pueden ser objeto de una crítica inmanente, al comparar su concreción con la pugna por el reconocimiento que las animó. Las estaciones de la filosofía del derecho hegeliana se reformulan entonces como libertad jurídica, libertad moral y libertad social, en el marco de una eticidad democrática (Honneth, 2014). Para no recaer en positivismo, Honneth ha de marcar distancia con lo existente, completando esta especie de trilogía su obra con una revisión del ideal socialista (Honneth, 2015).

Después de esta esquemática exposición de las teorías que Honneth ha ido componiendo en los últimos 35 años, podemos preguntarnos ya sobre la relación con la sociología de la educación.

\section{La teoría del reconocimiento de Honneth y la sociología de la educación}

El objeto de la sociología de la educación puede ser considerado de maneras distintas. La educación es un derecho, un sistema o un conjunto de cambios en las personas. Frecuentemente, la sociología de la educación reduce su objeto al análisis de un sistema social y, más aún, al estudio del dispositivo escolar, y todavía más, a la crítica del carácter ideológico del currículum. La perspectiva del reconocimiento tiene, en primer lugar, la virtualidad de recuperar un enfoque amplio, casi cenital, que pretende combinar lo descriptivo con lo normativo y lo general con lo particular, por lo que es adecuada para la triplicidad indicada en la noción de educación.

La aplicación de la teoría del reconocimiento a la sociología de la educación tiene que evitar los simplismos. Por ejemplo, identificar los modos de reconocimiento del amor, el derecho y la solidaridad o el reconocimiento de la aportación a lo colectivo, con los niveles educativos centrados en el cuidado (educación infantil y primaria), la socialización de la ciudadanía (secundaria) y la instrucción para el beneficio colectivo (secundaria postobligatoria o terciaria). El mismo Honneth ha defendido que la educación no coincide con un modo de reconocimiento concreto. En su «Réplica a la réplica» a Nancy Fraser se encuentra una breve mención al respecto, en la que afirma que «el sistema escolar estatal se integraría normativamente mediante dos ideas de reconocimiento social que compiten la una con la otra». (Honneth 2003: 293), y apela a los estudios de François Dubet, para quien en la educación se combinan un elemento democrático y otro meritocrático (Dubet 2007). Citaremos en extenso las propias palabras de Honneth en una entrevista que le realizamos en este año 2016 y que se ha publicado recientemente (Honneth 2017):

«La idea que en nuestras sociedades las escuelas están determinadas por dos normas que compiten mutuamente, la adopté del sociólogo francés Francois Dubet; este ha querido mostrar en una investigación muy bella que en las escuelas predomina tanto la norma de la "igualdad" como la norma del "rendimiento", sin que quede establecido de una vez.por todas de forma institucional cómo se deben relacionar la una con la otra. Esto me parece una idea altamente fructifera, que naturalmente se podría traducir con facilidad a mi teoría de reconocimiento: en nuestro sistema escolar se dirige a las alumnas y los alumnos o bien como a futuros ciudadanos, con la pretensión de que tengan las mismas posibilidades de participación y colaboración, o bien como a portadores de rendimientos en el mercado de trabajo, con todas las diferencias ya existentes en el correspondiente perfil del rendimiento. La misma distinción entre las dos formas de reconocimiento se puede describir también de manera que en el primer caso se comprende la enseñanza escolar como preparación a la formación de la voluntad democrática, mientras que en el segundo caso la enseñanza es entendida como cualificación pedagógica para el mercado laboral capitalista; en un caso, la escuela forma la bisagra institucional entre la socialización familiar y el Estado democrático de derecho, en el otro caso es la bisagra institucional entre la familia y el sistema económico. Sobre cuál de las dos principios de reconocimiento domina en la escuela en cada momento, deciden siempre, tal como lo veo yo, las disputas político-morales dentro del ámbito público acerca del 
papel y de la tarea de la educación escolar; y, según mi impresión, no bay duda de que hoy en día, en la ejecución de la llamada trasformación "neoliberal" del capitalismo occidental, el segundo principio de reconocimiento ha alcanzado el predominio, fuertemente apoyado por parte de los padres de las capas sociales más altas que asi esperan ventajas competitivas para sus hijas e hijos en el mercado laboral. Por regla general quizá se podría decir que en épocas de reformas democráticas y de un mercado contraido siempre alcanza el predominio en el sistema escolar el primer principio de reconocimiento -como en los años 60 y 70-, mientras que en épocas del retroceso se impone el principio de rendimiento. Pero desde luego bay que tener cuidado con tales generalizaciones históricas».

Al hilo de la exposición del epígrafe 2, se entenderá fácilmente que una primera gran consecuencia de la aplicación de la teoría del reconocimiento a la sociología de la educación es la superación de las perspectivas de la acción comunicativa, que han gozado de gran predicamento. La teoría del reconocimiento cercena el vínculo entre universalidad discursiva y primacía moral, que Habermas (2010) creyó ver ratificado en la aplicación que Lawrence Kohlberg hizo de las teorías de Piaget. Cortado ese nexo no con la navaja de Ockham, sino más bien con el estilete de Foucault y Bourdieu, tendría que disolverse aquella noche dialógica en la que todos los gatos son pardos, que hubiera dicho Hegel. Siguiendo con la entrevista:

«Es cierto que la teoria habermasiana de la comunicación tenía al principio, es decir desde los años 80, una especie de monopolio dentro de las ciencias de educación, pero la infuencia disminuyó notablemente cuando comenzaron a surgir dudas en la teoría de las etapas de desarrollo moral [Koblberg] que seguía a Piaget. En el fondo, de nuevo creció lentamente la comprensión sociológica de que tales modelos de etapas dicen poco sobre la motivación efectiva de la acción moral y además prácticamente no hacen justicia a las diferentes formas sociales de lo moral. A la sombra de tales dudas crecientes se efectuó entonces dentro de la pedagogía -y aqui solo puedo bablar de la situación alemana- una cierta pluralización».

Con esta pluralización a la que se refiere Honneth en la entrevista, ganó influencia la teoría de Luhmann y la propia teoría del reconocimiento.

En el primer caso, la teoría sistémica de Luhmann (2002) recobró importancia en la reflexión educativa en el contexto germanófono por la posibilidad de relación con la teoría constructivista del aprendizaje (a título de ejemplo, véase Arnold; Nittel, 2015) y por la menos relevante «marcha triunfal de corta duración», en expresión de Honneth en la misma entrevista, con la investigación del cerebro (véase también: Alheit; Dausien, 2007).

En el segundo caso, el propio Honneth (2016b) explica:

«De todas formas, como consecuencia de esta pluralización también la teoría del reconocimiento ganó importancia de forma notable en las ciencias de educación, sobre todo, si lo contemplo de manera adecuada, en la pedagogía general, pero también en la investigación empírica sobre la escuela. En la medida en la que soy capaz de abarcar este interesante campo, se realizan desde hace algunos años investigaciones fascinantes sobre la negociación de reconocimiento entre profesores y alumnos por un lado y entre diferentes grupos de alumnos por el otro: en ello se sigue naturalmente la idea de que tales conflictos de reconocimiento se efectúan al principio por debajo del umbral de la articulación verbal y que giran alrededor de definiciones y atribuciones de "rendimiento", "competencia" y "éxito". Así se hace visible de repente la enseñanza escolar e incluso toda escuela como institución un trasfondo hasta ahora desatendido en buena medida, a saber la pugna de las personas implicadas-profesorado, alumnado y, lamentablemente también, padres y madres-por disponer de la autoridad para establecer criterios de rendimiento, objetivos de la enseñanza e incluso las tareas más generales de la escuela como 


\title{
Mauricio Rebelo Martins, Francesc J. Hernàndez y Benno Herzog. La sociología de la educación... \\ RASE 2017. Vol. 10. N. ${ }^{\circ}$ 1: Págs. 80-89 \\ doi: http://dx.doi.org/10.7203/RASE.10.1.9451
}

\begin{abstract}
tal. Todo ello está cambiando constantemente, pero solo se puede apreciar cuando se deja el nivel de la comunicación oficial y se enfoca al intercambio de gestos corporales, de señales silenciosas y del currículo "oculto", como se denomina en las investigaciones».
\end{abstract}

Los ejemplos citados por Honneth permiten replantear algunos temas clásicos de la nueva sociología de la educación con un nuevo instrumental teórico. Por citar solo dos ejemplos relacionados con la perspectiva de género, los estudios de Elizabeth Grugeon (1995) sobre la «cultura» del patio de recreo y sus implicaciones de género, entre ellas el papel de las cancioncillas escolares de las niñas como rito iniciático o los trabajos de Julia Stanley (1995), que suponen una revisión de la obra de Willis a propósito del mito y la realidad de la alumna «apacible» (la «niña diez», digamos), se pueden reformular desde la pugna por el reconocimiento de las niñas en el contexto escolar discriminatorio. No hay que olvidar que el tema de los apodos que los discentes ponen a los docentes puede guardar relación con una práctica de resistencia ya indicada por Max Weber (1964: 685): «la “murmuración” de los trabajadores que nos revela la ética del antiguo Oriente: la desaprobación moral de la conducta mantenida por el jefe», que Honneth encuentra paradigmática de una pretensión de reconocimiento de sujetos ${ }^{4}$ que no pueden acceder al espacio público habermasiano (porque lo tiene vedado por la «autoridad pedagógica», que dirían Bourdieu y Passeron). Pero tampoco hay que caer en el simplismo de entender el reconocimiento como mero ideal ético-moral, es decir, «cosificarlo», en vez de destacar su carácter procesual en la teoría del reconocimiento (cf. Klinskisch, 2015) y analizar sus condiciones sociales y políticas.

En esta misma línea, hemos podido ofrecer análisis sobre prácticas educativas de diversos niveles relacionadas con el mismo modo de reconocimiento, el de la solidaridad, centrándonos en diversas manifestaciones estéticas, a saber, una práctica de teatro escolar vinculada con la ciudad (Muntanyola; Muntanyola, 2011) y un estudio sobre los PHD Cómics, elaborados en el contexto de estudios de doctorado (cf. Hernàndez; Herzog, 2015, caps. 11.3 y 11.4).

Otro vínculo entre la teoría del reconocimiento y la sociología de la educación tiene que ver con las tendencias privatizadoras, que se registran a escala planetaria (Arnove; Torres; Franz, 2016). En la conferencia de Honneth sobre «Educación y espacio público democrático» (Honneth, 2012), uno de los pocos textos en los que explícitamente aborda la cuestión educativa, reclama el desarrollo de formas de comunicación en la enseñanza «en las que las diferencias culturales no solo sean aceptadas como jugando, sino que puedan ser comprendidas como un enriquecimiento mutuo». (Honneth, 2012: 12). Sin embargo, puntualizamos nosotros, en muchos Estados occidentales la ampliación del sistema privado de educación, a parte de intereses económicos obvios, se justifica justamente por el deseo de una pluralidad en las formas de educación (recuerdese que los dos principios que determinaron la ley No Child Left Behind de Bush fueron testing y choising, exactamente los mismo que transplantó Wert con la LOMCE). En la entrevista citada, tuvimos la oportunidad de preguntar a Honneth lo siguiente: «¿Describiría esta forma de pluralidad como una patología social del sistema educativo que socava el potencial integrador de las instituciones públicas de educación?». La respuesta de Honneth resulta contundente y merece la pena ser transcrita en su integridad:

«Mi respuesta a ello es un "si" rotundo. Por varias razones soy muy escéptico respecto de la afirmación de que la ampliación de un sistema de escuelas privadas sirva para la pluralización de estilos educa-

4 Sin duda el mejor tratamiento literario de este tema lo ofrece Juan Rulfo con su Pedro Páramo, cuyo protagonista, Juan Preciado, confiesa a su vecina de tumba Dorotea «Me mataron los murmullos» (frag. 35). Precisamente así, Los murmullos se titulaba un relato previo de Rulfo (Cf. Galaviz, 2003). 
tivos y, con ello, para la multiplicación de valores culturales. Primero hay que tener presente que las ideas de los valores y los objetivos educativos que se cultivan especialmente en tales escuelas privadas la mayoría de las veces son la expresión de ideas que tienen los padres sobre el futuro ético de sus hijos; en este punto, en la elección de la escuela privada, solo raras veces resulta efectivo el deseo autónomo de un niño o un joven; más bien son los padres quienes deciden qué tipo de educación axiológica deben recibir sus hijos en la enseñanza escolar. En este sentido la escuela privada aumenta las posibilidades de los padres de decidir sobre las preferencias futuras y las orientaciones axiológicas de sus hijos; este poder de socialización de padres ya es lo suficientemente alto y no debería ser aumentado mediante la autorización estatal de escuelas privadas. Segundo e igualmente evidente es que un sistema de escuelas privadas está acompañado por el peligro de que la injusticia educativa existente en nuestras sociedades siga aumentando; y es que la asistencia a tales escuelas que no reciben financiación pública, por regla general, es muy cara asi que solo los hijos de padres pudientes pueden "disfrutar" de ellas. Además bay que tener en cuenta que aquellos padres que ban colocado a sus hijos en el "puerto" seguro de una escuela privada ya no van a abogar abiertamente por los asuntos del sistema escolar público; estas escuelas pierden, por lo tanto, un grupo importante de patrocinadores potentes y poderosos. También se podría decir quizá que cuantas más escuelas privadas, tanto menor es el compromiso del espacio público democrático de disponer de un sistema sólido, lo más justo posible de educación pública. Y finalmente, tercero, el Estado de derecho abandona casi el único medio que tiene para crear en la futura generación las actitudes y orientaciones valorativas necesarias para la propia reproducción; solo las escuelas públicas se pueden ocupar de una forma generalmente controlada (mediante regulaciones para la participación y mediante la elección de métodos de enseñanza) para que sus alumnos adquieran los conocimientos y las orientaciones indispensables para una participación activa en la formación democrática de la voluntad. Como se ve, respecto a la propuesta que se extiende hoy en día de ampliar aun más el sistema de escuelas privadas, tengo reparos, los mayores de ellos proceden de mis convicciones democráticas».

Adviértase la importancia que Honneth atribuye a las instituciones en la reproducción de la democracia, o mejor de una eticidad democrática.

Comentadas estas relaciones entre la teoría del reconocimiento y la sociología de la educación, abordaremos ahora, a manera de conclusión, algunas reflexiones más generales.

\section{Conclusiones}

En el epígrafe anterior, hemos comentado ejemplos en los que se puede apreciar la virtualidad de la teoría del reconocimiento para la sociología de la educación. Ahora se trata de aportar algunas conclusiones de carácter todavía más general.

Antes que nada hemos de comentar que aunque la teoría del reconocimiento ha sido formulada y reformulada a lo largo de 35 años, todavía tiene abiertos diversos frentes y está exigida de desarrollo. En relación con el tema de la educación, no es menor la cuestión de que Honneth haya eludido en la fundamentación de su teorización la obra capital de Hegel, a saber, la Fenomenología del espiritu (y su versión abstracta, la Ciencia de la lógica) cuando resulta indudable que el tema del reconocimiento atraviesa toda la obra (recuérdese como botón de muestra la dialéctica del amo y el esclavo). La Fenomenología del espíritu, o «ciencia de la experiencia de la conciencia» se entiende como un proceso formativo, en el sentido del término alemán Bildung. Y esta interpretación está más que acreditada por el gran impacto pedagógico que tuvo la derecha hegeliana, que convirtió el decurso del espíritu en pauta de ciencia histórica (Dilthey) y programa formativo (Krause). No se puede entender la Escuela Nueva, por ejemplo, sin el nexo con aquella interpretación formativa de Hegel, pero Honneth no ha dado ese paso. Si adoptáramos esa perspectiva dialéctica, cobraría 
pleno sentido la cuestión suscitada por Gregor Sauerwald (2012): «ilucha por el reconocimiento y/o proceso de aprendizaje?». Aunque esta pregunta, y con vista a los procesos mutilados de aprendizaje, también se puede formular con Eva Klinkisch (2015) como «ireconocimiento o semiformación?» (entendiendo «semiformación» en el sentido de Adorno, como una formación que se cree formación pero que solo es superficial y tiene como objetivo la adaptación y la funcionalidad.

Esta cuestión suscita la de los rendimientos que la teoría de educación o la del aprendizaje pueden ofrecer a la propia teoría del reconocimiento, lo que estaba apuntado ya en las menciones anteriores a Dubet. De nuevo vamos a recoger una declaración extensa de Honneth, cuya importancia glosaremos después. Preguntado por su experiencia de profesor, comenta:

\begin{abstract}
"Cuento todo esto para dejar claro que el papel del reconocimiento en la enseñanza y en las clases universitarias brotó en mi ya de una forma preteórica, por asi decir, y desde luego solo de una forma implicita en mis propias carnes. Desde entonces intento tomar en consideración aquello de lo que tuve experiencia en aquella época, tanto la experiencia positiva, como la negativa. Sobre todo me be puesto como tarea tomar de la manera más seria posible cada pregunta que se plantea en el aula, esto es, repetirla una vez más dándole la forma de interpretación más benévola para dar a entender al estudiante respectivo que su palabra cuenta y que tiene el derecho a una respuesta. Además presto mucha atención para conocer el trasfondo cultural de cada estudiante. En las tutorias siempre pregunto, a fin de indicar de este modo que soy consciente de las dificultades de la adaptación a un sistema cultural totalmente diferente. No bay más que decir, salvo dar a entender desde el principio a cada participante de una clase universitaria que su palabra tiene peso porque podria abrir perspectivas interesantes y alternativas de una interpretación creativa; es decir, hay que conceder algo asi como un reconocimiento "anticipatorio" que indique la suposición de que se trata de un estudiante con mucho talento. Naturalmente, en este asunto también resulta importante no hacer ninguna diferencia entre los géneros o por la pertenencia cultural, e incluso intentar sacar lo mejor de las manifestaciones de los representantes de una minoría».
\end{abstract}

Adviértase que la noción de «reconocimiento anticipatorio» representa una interesante aportación por dos razones. Por una parte, conecta el tema clásico del efecto Pigmalión, es decir, la prelación de las expectativas respecto del rendimiento. Por otra parte, respecto a su clasificación de modos de reconocimiento, la noción apunta en la dirección de un estudio de las condiciones previas del reconocimiento, esto es, de las condiciones sociales que determinan lo que es adecuado y que naturalmente son el objeto de la crítica social.

Apuntaremos dos cuestiones más que merecen ulteriores estudios. Por una parte, los rendimientos para el análisis sociológico de la educación de los trabajos realizados en el seno de la teoría del reconocimiento a propósito de realidades discursivas y no discursivas (o si se prefiere: lenguajes no verbales o que no acceden al espacio público) (sobre esto, cf. Herzog, 2016). Por otra, en la estela de la teoría del reconocimiento, el afinamiento de la descripción de las formas de desprecio (cf., por ejemplo, Jaeggi, 2016), que puede aportar nueva luz a los análisis de las relaciones educativas.

Como conclusión, la teoría del reconocimiento ha realizado aportes significativos a una sociología de la educación que esté a la altura de la teoría sociológica más reciente y, al mismo tiempo, puede beneficiarse de múltiples aportaciones sobre la educación y el aprendizaje, algunas de las cuales proceden de la sociología de la educación. 


\section{Referencias bibliográficas}

Adorno, Theodor W. (2011). Dialéctica negativa. La jerga de la autenticidad (Obra completa, VI). Madrid: Akal.

Adorno, Theodor W. (2014). Vorlesungen über Negative Dialektik. Fráncfort d. M.: Suhrkamp.

Alheit, Peter; Dausien, Bettina (2007): “La construcció biogràfica de la realitat". Index: En el curs de la vida. Xàtiva: CREC, pp. 93-114.

Arnold, Rolf; Nittel, Dieter (2015): “¿Del déficit tecnológico a la autotecnología?” en Francesc J. Hernàndez, Alicia Villar: Educación y biografías. Perspectivas pedagógicas y sociológicas actuales. Barcelona: Universitat Oberta de Cataluya.

Arnove, Robert F.; Torres, Carlos A.; Franz, Stephen (2016). Educación comparada. La dialéctica de lo globaly lo local. Valencia: Tirant lo Blanch.

Dubet, François (2007). "Injustices et reconnaissance” en Alain Callé (ed.): La quête de reconnaissance. Nouveau phénomène social. París: La decouverte.

Galaviz, Juan Manuel (2003). "De Los murmullos a Pedro Páramo”, en: Federico Campbell (ed.): La ficción de la memoria, México: Era; UNAM.

Fraser, Nancy; Honneth, Axel (2006). ¿Redistribución o reconocimiento? Madrid: Morata.

Grugeon, Elisabeth (1995). "Implicaciones del género en la cultura del patio de recreo" en Peter Woods, Martyn Hammersley: Género, cultura y etnia en la escuela, Madrid; Barcelona, MEC; Paidós.

Habermas, Jürgen (2010). Teoría de la acción comunicativa. Madrid: Trotta.

Hegel, Georg Wilhelm Friedrich (2009). Fenomenología del espiritu. Valencia: Pre-textos.

Hernàndez, Francesc J. (2009). "Educación en Hegel". Revista de la Asociación de Sociología de la Educación (RASE), 2 (3), 113-116.

Herzog, Benno (2016). Discourse Analysis as Social Critique. Discursive and Non-Discursive Realities in Critical Social Research. Londres: Palgrave; Macmillan.

Herzog, Benno; Hernàndez, Francesc J. (2012): “La noción de «lucha» en la teoría de reconocimiento de Axel Honneth. Sobre la posibilidad de subsanar el «déficit sociológico» de la Teoría Crítica con la ayuda del Análisis del Discurso". Politica y sociedad, 49, 609-623.

Herzog, Benno; Hernàndez, Francesc J. (2015). Estética del reconocimiento. València: Publicacions de la Universitat de València.

Honneth, Axel (1997). La lucha por el reconocimiento. Por una gramática moral de los conflictos sociales. Barcelona: Crítica Mondadori.

Honneth, Axel (1999). Suffering from Indeterminacy. An Attempt at Reactualization of Hegel's Pbilosopby of Right. Amsterdam: Spinoza Lectures.

Honneth, Axel (2001). Leiden an Unbestimmtheit. Eine Reaktualisierung der Hegelschen Rechtsphilosophie. Stuttgart: Reclam.

Honneth, Axel (2003). Unsichtbarkeit. Stationen einer Theorie der Intersubjektivität. Fráncfort d. M.: Suhrkamp. 
Honneth, Axel (2007). Reificación. Un estudio en la teoría del reconocimiento. Buenos Aires; Madrid: Katz.

Honneth, Axel (2009a). Crítica del poder. Fases en la reflexión de una Teoría Crítica de la sociedad. Madrid: Antonio Machado ed.

Honneth, Axel (2009b). Patologías de la razón. Historia y actualidad de la Teoría Crítica. Buenos Aires; Madrid: Katz.

Honneth, Axel (2009c). Crítica del agravio moral. Patologías de la sociedad contemporánea. México: Fondo de cultura económica.

Honneth, Axel (2010). The Pathologies of Individual Freedom: Hegel's Social Theory. Princenton: Princenton University Press.

Honneth, Axel (2011): “Conciencia moral y dominación de clase” en Francesc J. Hernàndez, Benno Herzog: La sociedad del desprecio. Madrid: Trotta.

Honneth, Axel (2012). "Erziehung und demokratische Öffentlichkeit. Ein vernachlässigtes Kapitel der politischen Philosophie”. Zeitschrift für Erriehungswissenschaft, 15 (3), 429-442.

Honneth, Axel (2014). El derecho de la libertad. Esbozo de una eticidad democrática. Madrid; Buenos Aires: Katz.

Honneth, Axel (2015). Die Idee des Sozialismus. Berlín: Suhrkamp.

Honneth, Axel (2016). Patologias de la libertad. Buenos Aires: Las cuarenta.

Honneth, Axel (2017). "La educación y la teoría del reconocimiento". Revista Educação \& Realidade, 42 (1), 395-406.

Honneth, Axel; Kemper, Peter; Klein, Richard (eds.) (2007). Bob Dylan. Ein Kongreß. Ergebnisse des internationalen Bob Dylan-Kongresses 2006 in Frankfurt am Main. Fráncfort d. M.: Suhrkamp.

Jaeggi, Rahel (2016). Entfremdung: Zur Aktualität eines sozialphilosophischen Problems. Berlín: Suhrkamp.

Klinkisch, Eva (2015). Habbildung oder Anerkennung? Perspektiven kritischer Bildung in der Gegenwart. Weinheim; Basel: Beltz Juventa.

Luhmann, Niklas (2002). Das Erziehungssystem der Gesellschaft. Fráncfort d. M.: Suhrkamp.

Marx, Karl (1978): "Crítica de la filosofía del Estado de Hegel”: Manuscritos de París. Anuarios francoalemanes (Obras de Marx y Engels, V). Barcelona: Crítica; Gijalbo.

Muntanyola, Josep; Muntanyola, Dafne (2011). "La sociología del espacio al encuentro de una arquitectura oculta en la educación”. Revista de la Asociciación de Sociología de la Educación (RASE), 4, 133-151.

Sauerwald, Gregor (2012). “Anerkennungskämpfe und/oder Lernprozesse?”. Manuscrito inédito.

Stanley, Julia (1995): "El sexo y la alumna tranquila" en Peter Woods, MartynHammersley: Género, cultura y etnia en la escuela. Madrid; Barcelona: MEC: Paidós.

Weber, Max (1964). Economía y sociedad. México: Fondo de cultura económica. 